\title{
Impact of Visceral Fat on Survival and Metastasis of Stage III Colorectal Cancer
}

\author{
Jung Won Park', Soo Yeon Chang'2, Joon Seok Lim³, Soo Jung Park ${ }^{1}$, Jae Jun Park',4, Jae Hee Cheon¹, Won Ho \\ $\mathrm{Kim}^{1}$, and Tae $\| \mathrm{Kim}^{1,4,5}$ \\ ${ }^{1}$ Department of Internal Medicine and Institute of Gastroenterology, Yonsei University College of Medicine, ${ }^{2}$ Health Promotion Center, \\ Severance Hospital, Yonsei University College of Medicine, ${ }^{3}$ Department of Radiology, Yonsei University College of Medicine, ${ }^{4}$ Yonsei \\ Cancer Prevention Center, Severance Hospital, Yonsei University College of Medicine, and ${ }^{5}$ Brain Korea 21 PLUS Project for Medical \\ Science, Yonsei University College of Medicine, Seoul, Korea
}

\section{Article Info}

Received August 21, 2020

Revised January 8, 2021

Accepted January 19, 2021

Published online July 28, 2021

\section{Corresponding Author}

Tae II Kim

ORCID https://orcid.org/0000-0003-4807-890X

E-mail taeilkim@yuhs.ac
Background/Aims: Previous studies have investigated the relationship between visceral obesity and the risk of colorectal tumors. Visceral obesity may affect the outcome of colorectal cancer $(\mathrm{CRC})$, including survival and metastasis. We investigated the associations between visceral adipose tissue and oncologic outcomes in stage III CRC.

Methods: Four hundred seventy-two patients with stage III CRC were identified. Subcutaneous and visceral adipose tissue areas were measured volumetrically via computed tomography for each patient at different levels of the lumbar spine. After adjusting for age, sex, and other clinical factors, the effects of visceral adipose tissue area on mortality and recurrence were assessed using Cox proportional hazard regression.

Results: In univariate and multivariate analyses, a higher visceral adipose tissue to total adipose tissue (VT) ratio (hazard ratio [HR], 1.041; 95\% Cl, 1.008 to 1.075; $p=0.015$ ) and higher visceral adipose tissue to subcutaneous adipose tissue (VS) ratio (HR, 1.016; 95\% Cl, 1.005 to 1.028; $p=0.006$ ) were both associated with poor CRC-specific survival. Interestingly, in the evaluation of each site of recurrence, a higher VT ratio (HR, 1.069;95\% $\mathrm{Cl}, 1.010$ to $1.131 ; \mathrm{p}=0.020)$ and higher VS ratio (HR, 1.024; $95 \% \mathrm{Cl}, 1.003$ to $1.045 ; \mathrm{p}=0.023)$ were both related to a higher risk of peritoneal seeding and tumor recurrence. The VT ratio at the L3-L4 level was significantly associated with a higher risk of peritoneal seeding and tumor recurrence $(\mathrm{HR}, 4.969 ; 95 \% \mathrm{Cl}, 1.303$ to 18.949; $p=0.019$ ), while other levels showed no such relationship.

Conclusions: Visceral obesity is closely related to increased risks of CRC-specific mortality and peritoneal seeding metastasis in stage III CRC patients. (Gut Liver 2022;16:53-61)

Key Words: Obesity; Fat; Visceral; Colorectal neoplasms; Outcome

\section{INTRODUCTION}

Obesity and cancer are the two most serious emerging health problems, and their interrelationship has also been known. ${ }^{1}$ Obesity is a well-known risk factor for the development of colorectal cancer (CRC) and is also associated with higher risks of postoperative complication rates and recurrence after adjuvant chemotherapy. ${ }^{2,3}$ Although the underlying mechanisms of their interaction remain unclear, recent data suggests an important role of visceral fat in this relationship. ${ }^{4,5}$ Visceral adipose tissue (VAT) has higher hormonal and metabolic activities than subcutaneous adipose tissue (SAT). ${ }^{6}$ Visceral adipocyte-induced insulin-like growth factor, inflammatory adipokines like interleukin- 6 and tumor necrosis factor- $\alpha$, and angiogenic factors are reported as mediators related with the tumorigenesis of obesity-related tumors. ${ }^{7-9}$ Furthermore, insulin resistance, commonly found in obesity, results in compensatory hyperinsulinemia. As key growth factors for colonic epithelial cells, insulin and C-peptide are well known mitogens of tumor cell growth. ${ }^{10-12}$

Previous studies have revealed the relationship between 
CRC and visceral obesity (VO). However, most studies have focused on the risk of complications after surgery, rather than recurrence or survival of CRC patients. ${ }^{13-16} \mathrm{~A}$ few studies have shown the oncologic outcomes of CRC in viscerally obese patients; however, the results and conclusions of such studies vary. Some studies have shown that $\mathrm{VO}$ is related to poor progression-free survival and poor response to adjuvant chemotherapy ${ }^{17-19}$ while other studies have argued that there is no convincing relationship between VO and survival or tumor stage of CRC. ${ }^{20,21}$ The role of VAT in CRC progression and recurrence remains to be elucidated and the numbers of studies concerning the association between metastasis and recurrence of CRC and VAT are very few.

We investigated the associations between VAT and oncologic outcomes, including overall survival, CRC-specific survival, progression-free survival, and metastasis sites, such as the liver, lung, or peritoneum, in stage III CRC.

\section{MATERIALS AND METHODS}

\section{Patients and data collection}

The medical records of patients with colon or rectal cancer, who had undergone surgical bowel resection on account of CRC, and who had been seen at Severance Hospital, Yonsei University, Seoul, Korea, between January 1, 2007, and December 31, 2009, were identified. Among those identified, patients with metastasis at the time of diagnosis were excluded, as they were likely to have undergone significant weight loss, thus lowering the reliability of estimates on obesity and fat distribution. Patients were also excluded from the study if preoperative abdominal computed tomography (CT) could not be evaluated or if patients were diagnosed with any other type of cancer prior to the diagnosis of CRC. Finally, 472 patients diagnosed with stage III CRC were included.

Patient demographics and clinical characteristics, including age at diagnosis, gender, tumor stage at diagnosis and surgical diagnosis, and underlying medical conditions were obtained from medical records. Data regarding the history of use of drugs, family history of CRC, smoking history, drinking history, date of death and recurrence, cause of death, and received treatment were also obtained. The date of CRC diagnosis was defined as the day of pathologic diagnosis. Deaths were identified through medical records, and cause of death was determined in all cases. Height and weight recorded before surgery were used to calculate body mass index (BMI; weight in $\mathrm{kg}$ divided by height in $\mathrm{m}^{2}$ ). BMI was stratified into "underweight" (BMI $<18.5 \mathrm{~kg} /$ $\mathrm{m}^{2}$ ), "normal" (BMI 18.5-24.9 kg/m²), "overweight" (BMI
$25.0-29.9 \mathrm{~kg} / \mathrm{m}^{2}$ ), and "obese" (BMI $\geq 30.0 \mathrm{~kg} / \mathrm{m}^{2}$ ) based on the World Health Organization BMI classification. ${ }^{22}$

The Institutional Review Board of Severance Hospital, Yonsei University, Seoul, Korea, approved this study in accordance with the Declaration of Helsinki, and IRB review and informed consent were exempted after review of study design.

\section{Measurements of abdominal adipose tissue}

Preoperative abdomen CT (Siemens Medical Systems, Forchheim, Germany) was performed on all patients. VAT and SAT areas were retrospectively measured from three axial slices at the L3-L4, L4-L5, and L5-S1 intervertebral space. The CT attenuation level was set between -190 to -30 Hounsfield units, regions of adipose tissue were delineated, and areas of total adipose tissue, VAT, and SAT were calculated. The VAT area referred to the intra-abdominal fat bound by the parietal peritoneum or transversalis fascia, excluding the vertebral column and paraspinal muscles (Supplementary Fig. 1A). SAT area was the fat superficial to the abdominal and back muscles (Supplementary Fig. 1B). Free hand-driving mode program was used to measure fat volume. Automated fat segmentation was performed after adjusting the boundaries for subcutaneous and visceral compartments at each level. Then, we manipulated the fat segments to either include or exclude focal regions as required. A skilled radiologist, blinded to the patient's data, measured all data.

The VAT to SAT ratio (VS ratio) and VAT to TAT ratio (VT ratio) were calculated to provide a single representative measure of abdominal fat.

\section{Assessment of CRC staging and treatment}

All patients with pathologically confirmed CRC were evaluated for appropriate staging based on 7th version of the American Joint Committee on Cancer TNM staging system. Treatment modality was determined based on the tumor extent and location at diagnosis and recurrence during follow-up. According to the National Comprehensive Cancer Network guideline, patients with locally advanced stage III CRC or recurrent advanced tumors with resectable metastatic lesions were treated by surgical resection, followed by adjuvant chemotherapy (FOLFOX regimen). Some patients with recurrent advanced tumors with metastasis were treated by neoadjuvant chemotherapy (FOLFIRI or FOLFOX with cetuximab or bevacizumab, based on primary tumor location and the result of KRAS mutation), followed by surgery. In case of rectal cancer, preoperative chemoradiation or postoperative radiation were added. Recurrent unresectable metastatic CRC were treated by palliative chemotherapy (FOFIRI or FOLFOX 
regimens) with target agent, such as cetuximab or bevacizumab, according to primary tumor location and the result of KRAS mutation analysis.

\section{Statistical analysis}

Descriptive statistics are presented as absolute (n) and relative (\%) frequencies for categorical variables, and median or mean \pm standard deviation for quantitative variables. In comparison of covariates between non-

Table 1. Patient Demographics and Baseline Clinical Characteristics ( $n=472$ )

\begin{tabular}{|c|c|c|c|c|}
\hline Variable & Total $(n=472)$ & $\begin{array}{c}\text { Non-overweight } \\
\left(\mathrm{BMI}<25 \mathrm{~kg} / \mathrm{m}^{2}\right)(\mathrm{n}=318)\end{array}$ & $\begin{array}{c}\text { Overweight } \\
\left(\mathrm{BMI} \geq 25 \mathrm{~kg} / \mathrm{m}^{2}\right)(\mathrm{n}=154)\end{array}$ & $\mathrm{p}$-value \\
\hline Age, yr & $59(26-86)$ & $59(26-86)$ & $58(29-80)$ & 0.355 \\
\hline Male sex & $272(57.6)$ & $177(55.7)$ & 95 (61.7) & 0.214 \\
\hline Smoking status & & & & 0.836 \\
\hline Current smoker & $285(60.4)$ & $191(60.1)$ & 94 (61.0) & \\
\hline Former smoker & 91 (19.3) & $60(18.9)$ & $31(20.1)$ & \\
\hline Never smoker & 96 (20.3) & $67(21.1)$ & 29 (18.8) & \\
\hline Daily drinker & $39(8.3)$ & $28(8.8)$ & $11(7.1)$ & 0.539 \\
\hline Family history of colorectal cancer & $35(7.4)$ & $23(7.2)$ & $12(7.8)$ & 0.828 \\
\hline \multicolumn{5}{|l|}{ Chronic disease and medication } \\
\hline Hypertension & 159 (33.7) & $103(32.4)$ & $56(36.4)$ & 0.392 \\
\hline Diabetes mellitus & 81 (17.2) & $56(17.6)$ & 25 (16.2) & 0.710 \\
\hline Regular use of metformin & $44(9.3)$ & $32(10.1)$ & $12(7.8)$ & 0.426 \\
\hline Regular use of low-dose aspirin & $94(19.9)$ & 58 (18.2) & $36(23.4)$ & 0.190 \\
\hline Fatty liver & 45 (9.5) & $20(6.3)$ & $25(16.2)$ & 0.001 \\
\hline Cancer location & & & & 0.545 \\
\hline Colon & $285(60.4)$ & $189(59.4)$ & 96 (62.3) & \\
\hline Rectum & $187(39.6)$ & $129(40.6)$ & $56(37.7)$ & \\
\hline T-stage & & & & 0.090 \\
\hline Tis & $2(0.4)$ & 0 & $2(1.3)$ & \\
\hline $\mathrm{T} 1$ & $19(4.0)$ & $11(3.5)$ & $8(5.2)$ & \\
\hline T2 & $46(9.7)$ & $28(8.8)$ & $18(11.7)$ & \\
\hline T3 & 346 (73.3) & 234 (73.5) & $112(72.7)$ & \\
\hline $\mathrm{T} 4$ & 59 (12.5) & 45 (14.2) & $14(9.1)$ & \\
\hline $\mathrm{N}$-stage & & & & 0.796 \\
\hline NO & $1(0.2)$ & $1(0.3)$ & 0 & \\
\hline $\mathrm{N} 1 \mathrm{a}$ & $177(37.5)$ & 123 (38.7) & $54(35.1)$ & \\
\hline $\mathrm{N} 1 \mathrm{~b}$ & 150 (31.8) & 96 (30.2) & $54(35.1)$ & \\
\hline $\mathrm{N} 1 \mathrm{c}$ & 0 & 0 & 0 & \\
\hline $\mathrm{N} 2 \mathrm{a}$ & 92 (19.5) & $63(19.8)$ & 29 (18.8) & \\
\hline $\mathrm{N} 2 \mathrm{~b}$ & $52(11.0)$ & $35(11.0)$ & $17(11.0)$ & \\
\hline Histologic type & & & & 0.497 \\
\hline Well differentiated & $45(9.5)$ & $27(8.5)$ & $18(11.7)$ & \\
\hline Moderately differentiated & $388(82.2)$ & 265 (83.3) & $123(79.9)$ & \\
\hline Poorly/undifferentiated & $22(4.7)$ & $14(4.4)$ & $8(5.2)$ & \\
\hline Unknown & $17(3.6)$ & $12(3.8)$ & $5(3.2)$ & \\
\hline \multicolumn{5}{|l|}{ Treatment } \\
\hline Neoadjuvant CCRT & $32(6.8)$ & $23(7.2)$ & $9(5.8)$ & 0.574 \\
\hline Adjuvant CTx & 460 (97.5) & 309 (97.2) & $151(98.1)$ & 0.568 \\
\hline Adjuvant CCRT & $95(20.1)$ & 63 (19.8) & 32 (20.8) & 0.806 \\
\hline Postoperative RT & $1(0.2)$ & 0 & $1(0.7)$ & 0.150 \\
\hline \multicolumn{5}{|l|}{ Measures of obesity* } \\
\hline VAT area, $\mathrm{cm}^{2}$ & $66.20 \pm 30.57$ & $57.62 \pm 26.53$ & $83.89 \pm 30.85$ & $<0.001$ \\
\hline SAT area, $\mathrm{cm}^{2}$ & $142.46 \pm 63.92$ & $125.25 \pm 55.18$ & $178.01 \pm 66.22$ & $<0.001$ \\
\hline VAT to TAT ratio & $32.46 \pm 9.81$ & $32.32 \pm 9.89$ & $32.76 \pm 9.65$ & 0.634 \\
\hline VAT to SAT ratio & $51.42 \pm 23.60$ & $51.13 \pm 23.56$ & $52.04 \pm 23.74$ & 0.696 \\
\hline
\end{tabular}

Data are presented as median (range), number (\%), or mean \pm SD.

BMI, body mass index; CCRT, chemoradiation; CTx, chemotherapy; RT, radiation therapy; VAT, visceral adipose tissue; SAT, subcutaneous adipose tissue; TAT, total adipose tissue.

*VAT area and SAT area: the average value for area at each of the three vertebral levels: L3-L4, L4-L5, and L5-S1. 
overweight and overweight groups, the Pearson chi-square test or Student $\mathrm{t}$-test were used for analyzing categorical or continuous data, respectively. Relationships between fat parameters and clinical outcomes were examined with Cox proportional hazards regression model, which was used to estimate hazard ratios (HRs) and 95\% confidence intervals (95\% CIs) after adjusting patient-related variables, including age at diagnosis, gender, and family history of CRC with medication, smoking, and alcohol history, when indicated. The Kaplan-Meier analysis was used for survival curves, which were compared by the log-rank test. Given well-known sex differences in fat distribution, we adjusted for sex in bivariate and multivariate analyses. Statistical analysis was performed using the software package SPSS 21.0 (IBM Corp., Armonk, NY, USA). A difference of $\mathrm{p}<0.05$ was considered statistically significant.

\section{RESULTS}

\section{Patients' demographics and clinical characteristics}

Four hundred and seventy-two patients diagnosed with stage III CRC underwent colorectal surgery. CT scans for evaluation of disease extent and staging were performed in all 472 patients. Among the enrolled, 154 patients were overweight, while 318 were not. Demographics and baseline clinical characteristics were mostly similar between the two groups, except for factors associated with fat distribution (Table 1). Median age at diagnosis was 59 years (range,
26 to 86 years), and 272 patients were men (57.6\%). As for age, gender, family history of cancer, chronic disease, and medication status, smoking history, and drinking history, there was no significant difference between the two groups. Tumor location, $\mathrm{T}$ and $\mathrm{N}$ stage, histologic type, and treatment type also showed no difference between the two groups. Average VAT area $\left(83.89 \pm 30.85 \mathrm{~cm}^{2}\right.$ vs $\left.57.62 \pm 26.53 \mathrm{~cm}^{2}, \mathrm{p}<0.001\right)$ and SAT area $(178.01 \pm 66.22$ $\mathrm{cm}^{2}$ vs $\left.125.25 \pm 55.18 \mathrm{~cm}^{2}, \mathrm{p}<0.001\right)$ were both significantly higher in the overweight group than in the non-overweight group. However, the VT and VS ratios did not show significant differences between the two groups.

\section{Fat distribution and survival analysis}

Among the entire cohort, there were total of 111 deaths, of which 67 cases were CRC-related. There were 114 cases of recurrence, with the lung being the most frequent site of recurrence ( 34 cases), followed by the liver and peritoneum, with 33 and 25 cases of recurrence, respectively. The median follow-up duration was 60 months (range, 12 to 84 months).

Overall survival and CRC-specific survival were investigated according to the obesity index (Tables 2 and 3). The Cox hazard regression analysis for overall survival showed that BMI was an independent risk factor for overall mortality. Higher BMI tended to display a lower risk of overall mortality in univariate analysis (HR, 0.939; 95\% CI, 0.884 to $0.998 ; \mathrm{p}=0.043$ ), and this result was consistent after adjusting for age at diagnosis, sex, and familial history

Table 2. Cox Hazard Regression Analysis for Overall Mortality According to Obesity Index

\begin{tabular}{lcccc}
\hline \multicolumn{1}{c}{ Obesity index } & Univariate HR $(95 \% \mathrm{Cl})$ & $\mathrm{p}$-value & Adjusted HR $(95 \% \mathrm{Cl})^{*}$ & $\mathrm{p}$-value \\
\hline BMI & $0.939(0.884-0.998)$ & 0.043 & $0.915(0.840-0.997)$ & 0.044 \\
Average VAT area & $1.000(0.994-1.006)$ & 0.888 & $1.003(0.995-1.011)$ & 0.506 \\
Average SAT area & $0.999(0.996-1.002)$ & 0.405 & $1.002(0.997-1.007)$ & 0.453 \\
VAT to TAT ratio & $1.020(1.001-1.039)$ & 0.043 & $1.022(0.997-1.049)$ & 0.091 \\
VAT to SAT ratio & $1.008(1.001-1.016)$ & 0.027 & $1.009(1.000-1.019)$ & 0.058 \\
\hline
\end{tabular}

$\mathrm{HR}$, hazard ratio; $\mathrm{Cl}$, confidence interval; BMI, body mass index; VAT, visceral adipose tissue; SAT, subcutaneous adipose tissue; TAT, total adipose tissue.

*Adjusted for age at diagnosis, sex, familial history of colorectal cancer.

Table 3. Cox Hazard Regression Analysis for Colorectal Cancer-Specific Mortality According to Obesity Index

\begin{tabular}{lcccc}
\hline \multicolumn{1}{c}{ Obesity index } & Univariate HR $(95 \% \mathrm{Cl})$ & $\mathrm{p}$-value & Adjusted HR (95\% Cl)* & $\mathrm{p}$-value \\
\hline BMI & $0.980(0.907-1.059)$ & 0.613 & $1.006(0.904-1.120)$ & 0.908 \\
Average VAT & $1.001(0.993-1.008)$ & 0.895 & $1.004(0.993-1.014)$ & 0.493 \\
Average SAT & $0.997(0.993-1.001)$ & 0.197 & $0.996(0.990-1.003)$ & 0.278 \\
VAT to TAT ratio & $1.031(1.006-1.056)$ & 0.014 & $1.041(1.008-1.075)$ & 0.015 \\
VAT to SAT ratio & $1.013(1.004-1.022)$ & 0.005 & $1.016(1.005-1.028)$ & 0.006 \\
\hline
\end{tabular}

HR, hazard ratio; $\mathrm{Cl}$, confidence interval; BMI, body mass index; VAT, visceral adipose tissue; SAT, subcutaneous adipose tissue; TAT, total adipose tissue.

*Adjusted for age at diagnosis, sex, familial history of colorectal cancer. 
of CRC (HR, 0.915; 95\% CI, 0.840 to 0.997; $\mathrm{p}=0.044$ ). Regarding CRC-specific survival analysis, BMI was not a significant independent factor associated with CRC-specific mortality (HR, 1.006; 95\% CI, 0.904 to 1.120 ; $\mathrm{p}=0.908$ ). Interestingly, neither average VAT nor SAT area was associated with overall mortality. Meanwhile, the VT ratio (HR, 1.020; 95\% CI, 1.001 to 1.039; $\mathrm{p}=0.043$ ) and VS ratio (HR, 1.008; 95\% CI, 1.001 to $1.016 ; \mathrm{p}=0.027$ ) were both related to higher overall mortality in univariate analysis. However, after adjustment for clinical factors, the results were not statistically significant. Nonetheless, VT and VS ratios were both independent predictors for CRC-specific mortality in stage III CRC after surgical resection (Table 3). In univariate analysis, both VT ratio (HR, 1.031; 95\% CI, 1.006 to 1.056; $\mathrm{p}=0.014)$ and VS ratio (HR, 1.013; 95\% CI, 1.004 to 1.022; $\mathrm{p}=0.005)$ were significantly associated with higher cancer-specific mortality; after adjustment of clinically relevant factors, the results maintained statistical significance (VT ratio: HR, 1.041; 95\% CI, 1.008 to $1.075 ; \mathrm{p}=0.015$ and VS ratio: HR, 1.016; 95\% CI, 1.005 to 1.028; $\mathrm{p}=0.006$ ).

Additionally, according to the VT ratios of each intervertebral level, overall survival, cancer-specific survival, and recurrence-free survival were analyzed. There were no significant results regarding the overall survival and recurrence-free survival (Supplementary Tables 1 and 2). However, all three VT ratios (at L3-L4, L4-L5, and L5-S1) showed significant results, as higher VT ratios were associated with higher cancer-specific mortality, in analyses both unadjusted and adjusted for clinically relevant factors (Supplementary Table 3).

\section{Fat distribution and recurrence}

Recurrence-free survival analysis was also performed according to obesity index (Table 4); however, there were no statistically significant results. BMI (HR, 0.978; 95\% CI, 0.898 to $1.065 ; \mathrm{p}=0.612$ ), VAT (HR, $0.995 ; 95 \% \mathrm{CI}, 0.986$ to $1.003 ; \mathrm{p}=0.234$ ), and VT ratio (HR, $0.989 ; 95 \% \mathrm{CI}, 0.963$ to 1.015; $\mathrm{p}=0.387$ ) were not associated with recurrence after treatment of stage III CRC. We performed additional analyses concerning the location of tumor recurrence. First, we investigated the relationship between fatty liver and liver recurrence; however, no significant association was found between these two factors (Supplementary Fig. 2). Liver and lung metastases according to obesity index were also evaluated separately; however, the analysis showed no significant results (Supplementary Tables 4 and 5). Analysis regarding peritoneal seeding recurrence provided interesting results (Table 5). In the multivariate analysis for

Table 4. Cox Hazard Regression Analysis for Recurrence According to Obesity Index

\begin{tabular}{lcccc}
\hline \multicolumn{1}{c}{ Obesity index } & Univariate HR $(95 \% \mathrm{Cl})$ & $\mathrm{p}$-value & Adjusted HR (95\% Cl)* & $\mathrm{p}$-value \\
\hline BMI & $0.971(0.915-1.031)$ & 0.342 & $0.978(0.898-1.065)$ & 0.612 \\
Average VAT & $0.996(0.990-1.003)$ & 0.249 & $0.995(0.986-1.003)$ & 0.234 \\
Average SAT & $0.999(0.996-1.002)$ & 0.372 & $1.002(0.997-1.007)$ & 0.541 \\
VAT to TAT ratio & $1.000(0.981-1.019)$ & 0.978 & $0.989(0.963-1.015)$ & 0.387 \\
VAT to SAT ratio & $1.000(0.992-1.008)$ & 0.976 & $0.996(0.986-1.006)$ & 0.446 \\
\hline
\end{tabular}

$\mathrm{HR}$, hazard ratio; $\mathrm{Cl}$, confidence interval; BMI, body mass index; VAT, visceral adipose tissue; SAT, subcutaneous adipose tissue; TAT, total adipose tissue.

*Adjusted for age at diagnosis, sex, familial history of colorectal cancer.

Table 5. Cox Hazard Regression Analysis for Peritoneal Seeding According to Obesity Index

\begin{tabular}{|c|c|c|c|c|}
\hline Obesity index & Univariate HR (95\% Cl) & p-value & Adjusted HR $(95 \% \mathrm{CI})^{*}$ & $\mathrm{p}$-value \\
\hline $\mathrm{BMI}$ & $0.884(0.780-1.002)$ & 0.054 & $0.877(0.774-0.994)$ & 0.040 \\
\hline Average VAT & $1.003(0.990-1.016)$ & 0.671 & $1.007(0.991-1.021)$ & 0.369 \\
\hline Average SAT & $0.999(0.992-1.006)$ & 0.797 & $0.997(0.990-1.004)$ & 0.393 \\
\hline VAT to TAT ratio & 1.017 (0.977-1.059) & 0.413 & $1.069(1.010-1.131)$ & 0.020 \\
\hline \multicolumn{5}{|c|}{ VAT to TAT ratio (top vs bottom 25th percentile) } \\
\hline$\leq 24.53 \%$ & 1.000 & & 1.000 & \\
\hline$>38.85 \%$ & $1.657(0.479-5.729)$ & 0.425 & $5.587(1.048-29.789)$ & 0.044 \\
\hline VAT to SAT ratio & $1.007(0.991-1.024)$ & 0.373 & $1.024(1.003-1.045)$ & 0.023 \\
\hline \multicolumn{5}{|c|}{ VAT to SAT ratio (top vs bottom 25th percentile) } \\
\hline$\leq 32.50 \%$ & 1.000 & & 1.000 & \\
\hline$>63.53 \%$ & $1.900(0.564-6.395)$ & 0.300 & $2.206(0.616-7.895)$ & 0.224 \\
\hline
\end{tabular}

$\mathrm{HR}$, hazard ratio; $\mathrm{Cl}$, confidence interval; BMI, body mass index; VAT, visceral adipose tissue; SAT, subcutaneous adipose tissue; TAT, total adipose tissue.

*Adjusted for age at diagnosis, sex, familial history of colorectal cancer. 

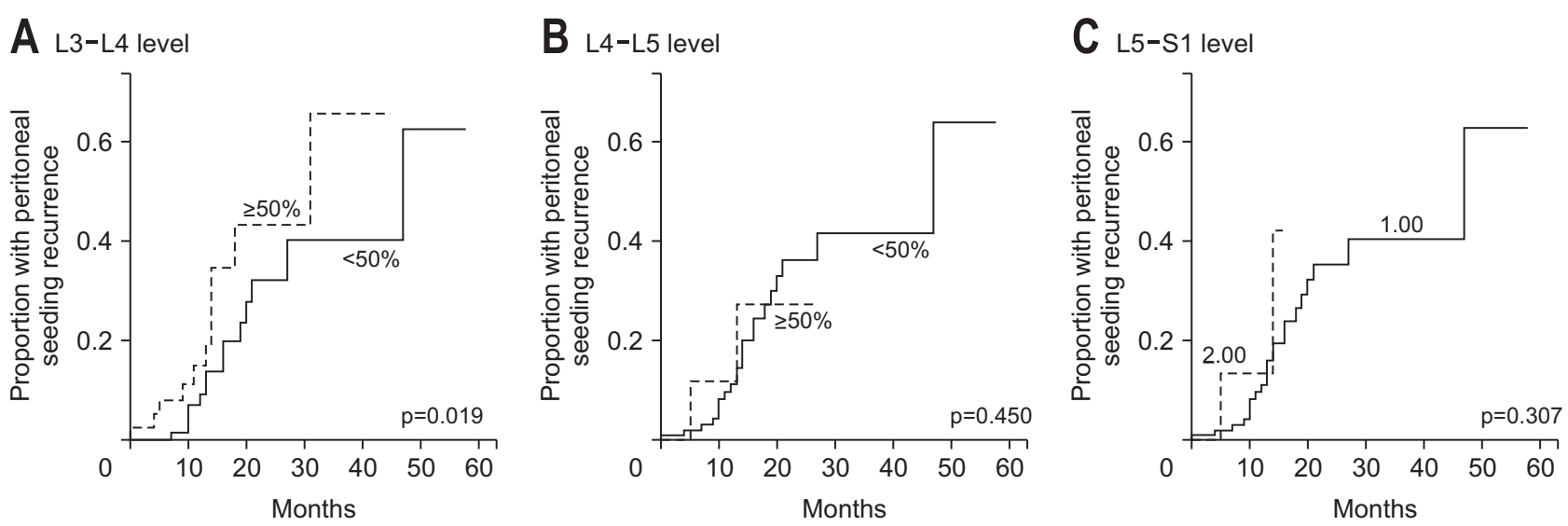

Fig. 1. Peritoneal seeding rate according to the visceral adipose tissue to total adipose tissue area ratio at each level of the lumbar spine in stage III colorectal cancer patients. (A) Visceral to total adipose tissue (VT) ratio at the L3-L4 level, (B) VT ratio at the L4-L5 level, (C) VT ratio at the L5-S1 level.

peritoneal seeding recurrence according to obesity index and after adjusting for clinically relevant factors, BMI, VT ratio, and VS ratio showed statistically significant results. Higher VT (HR, 1.069; 95\% CI, 1.010 to $1.131 ; \mathrm{p}=0.020$ ) and VS ratio (HR, 1.024; 95\% CI, 1.003 to $1.045 ; \mathrm{p}=0.023$ ) were both associated with a higher risk of peritoneal seeding recurrence, but higher BMI (HR, 0.877; 95\% CI, 0.774 to $0.994 ; \mathrm{p}=0.040$ ) was associated with a lower risk of peritoneal seeding recurrence. When VT ratio was divided into two groups by the highest and lowest 25 percentile number, VT ratio of the highest 25 percentile group had a 5 -time higher risk of peritoneal recurrence than the lowest 25 percentile group (HR, 5.587; 95\% CI, 1.048 to 29.789 ; $\mathrm{p}=0.044)$. However, results regarding VS ratio showed an irrelevant outcome (HR, 2.206; 95\% CI, 0.616 to 7.895 ; $\mathrm{p}=0.224$ ).

Since the VAT and SAT areas used in survival and recurrence analyses were the average values of three intervertebral levels, another subgroup analysis, comparing peritoneal recurrence risk of these three intervertebral levels, was performed. Peritoneal seeding recurrence rate according to VT ratio of each spine level in stage III CRC patients was best explained when applying the area of adipose tissue from the L3 to L4 intervertebral level (Fig. 1A). When VT ratio of the L3-L4 level was divided into two groups by 50 percentile number, VT ratio of higher than 50 percentile had almost a 5 -time higher risk of peritoneal recurrence than the lower than 50 percentile group (HR, 4.969; $95 \%$ CI, 1.303 to 18.949 ; $\mathrm{p}=0.019$ ). However, VT ratio of higher than 50 percentile at the L4-L5 level (HR, 0.514; 95\% CI, 0.091 to 2.892; $\mathrm{p}=0.450$ ) or L5-S1 level (HR, 2.617; $95 \% \mathrm{CI}, 0.413$ to $16.57 ; \mathrm{p}=0.307$ ) was not associated with a higher risk of peritoneal recurrence (Fig. 1B and C).

\section{DISCUSSION}

Obesity is known to be a risk factor for the development of several cancers, including CRC. However, the influence of VAT distribution on the outcomes of patients with CRC remains controversial. The objective of this study was to examine the outcome of CRC patients according to obesity indices including BMI, VAT area, SAT area, and their corresponding ratios.

Compared to SAT, VAT is known to be more biologically active and therefore related to obesity-related diseases including cancer. ${ }^{715,23}$ Most of VAT is distributed within the omentum, mesentery, and retroperitoneum. ${ }^{24}$ Then, we considered CT scan as a better method for measurement of fat because CT scan is mandatory examination before treatment of all newly diagnosed CRC. We focused on VAT distribution and its relative ratio to total adipose tissue or SAT distribution.

The most interesting finding in our study was that a higher VT ratio and a higher VS ratio were both associated with a higher risk of peritoneal seeding recurrence. To our knowledge, this is the first study to find a relationship between peritoneal seeding recurrence and visceral fat. Several studies have provided evidence of the significant contribution of VO to cancer development and aimed to understand the role of omental fat in intraperitoneal tumorigenesis. Using coculture system to simulate the crosstalk between adipocytes and cancer cells, Xiang et al. ${ }^{25}$ showed that omental adipocytes induced a significant increase of lipid uptake in gastric cancer cells, and enhanced their invasiveness. Similarly, another study using coculture of adipocyte and ovarian cancer cells demonstrated the direct transfer of lipids from adipocytes to cancer cells and an increase of tumor growth. ${ }^{26}$ In addition to the adipocytes, stimulation of peritoneal tumors is also associated 
with omental adipose tissue-derived stem cells (ASCs). Nowicka et al. ${ }^{27}$ reported that ASCs significantly promoted proliferation, migration, and resistance to chemotherapy and radiation in ovarian cancer cell lines by providing cancer cells with lactose and ATP generated in the glycolytic pathway. Another series of experiments using ASCs isolated from mice showed that cells isolated from the visceral fat of obese animals were able to increase the development of intraperitoneal tumors, whereas cells obtained from SAT lacked this activity. ${ }^{28}$

Along with these reports, the traditional "insulin hypothesis" has also been used to explain the relationship between obesity or visceral adiposity and CRC. ${ }^{11,29}$ Prospective studies have shown an increased risk of CRC in individuals with higher levels of postprandial insulin, ${ }^{30}$ C-peptide, ${ }^{31}$ and fasting glucose. ${ }^{30}$ However, Renehan et al. ${ }^{32}$ showed that not only the insulin/insulin-like growth factor molecular pathway, but also metabolic syndrome, aging, endogenous sex hormones, adiponectin, and leptin are all very complexly connected with inter-related regulation. Therefore, the insulin hypothesis, sex hormones and adiponectin, adipocyte-promoted lipid internalization of cancer cells, and ASCs affect and regulate each other in the process of peritoneal metastasis.

However, our assessment regarding peritoneal seeding recurrence and overall survival has shown that higher BMI was related to a lower risk of peritoneal seeding recurrence and overall mortality. In a recent review article regarding BMI, obesity, and their association with cardiovascular diseases, the authors claimed that BMI does not explain fat distribution or degree of VO. ${ }^{33}$ The metabolically healthy obese phenotype refers to a subgroup of obese individuals in which high BMI is associated with a healthy metabolic profile, characterized by high insulin sensitivity, favorable lipid profile, low VAT, and high lean mass. The "metabolically health" with healthy obese phenotype was not considered in our study, but this might have affected the results of our study, showing a low risk of peritoneal seeding and overall mortality in patients with higher BMI.

Our study has some limitations. First, given the retrospective nature of this study, the results require cautious interpretation. In addition, there is currently no established threshold of VAT area or VS ratio for defining VO. However, our findings might provide additional supportive evidence in this research area. Further, although our study includes a very heterogeneous patient population, it also includes a large number of subjects with stage III CRC, which improves the reliability of the results. Finally, the results of this study should be confirmed among the general population because patients of this study were from a tertiary medical institute.
In conclusion, $\mathrm{VO}$ is closely related to the increased risk of CRC-specific mortality and, most intriguingly, to higher risk of peritoneal seeding metastasis in patients with stage III CRC. These findings could be useful in strategy making process of diet and physical care for the patient group in actual clinical field. Further studies are needed to clarify the physiologic and biologic mechanisms mediating the link between visceral adiposity and CRC metastasis. The assimilation of quantitative measures of $\mathrm{VO}$ and corresponding clinical studies is needed to integrate these results with therapeutic and epidemiologic studies.

\section{CONFLICTS OF INTEREST}

S.J.P. and J.H.C. are editorial board members of the journal but were not involved in the peer reviewer selection, evaluation, or decision process of this article. No other potential conflicts of interest relevant to this article were reported.

\section{ACKNOWLEDGEMENTS}

This research was supported by a grant from the National R\&D Program for Cancer Control, Ministry of Health and Welfare, Republic of Korea (grant number: 1631020).

This abstract was presented in a poster session at Asian Pacific Digestive Week 2018 (APDW 2018), on November 15-18, 2018, in Seoul, Korea.

\section{AUTHOR CONTRIBUTIONS}

Study concept and design: T.I.K. Data acquisition: J.W.P., S.Y.C., S.J.P., J.J.P., J.H.C., J.S.L., W.H.K. Data analysis and interpretation: J.W.P., S.Y.C., J.S.L., T.I.K. Statistical analysis: J.W.P., S.Y.C. Drafting of the manuscript: J.W.P., S.Y.C. Critical revision of the manuscript: S.J.P., J.J.P., J.H.C., W.H.K., T.I.K.

\section{ORCID}

Jung Won Park https://orcid.org/0000-0003-1861-3975 Soo Yeon Chang https://orcid.org/0000-0003-3006-1640 Joon Seok Lim https://orcid.org/0000-0002-0334-5042 Soo Jung Park https://orcid.org/0000-0003-0699-6809 Jae Jun Park https://orcid.org/0000-0001-9974-1658 Jae Hee Cheon https://orcid.org/0000-0002-2282-8904 
Won Ho Kim

https://orcid.org/0000-0002-5682-9972

Tae Il Kim

\section{SUPPLEMENTARY MATERIALS}

Supplementary materials can be accessed at https://doi. org/10.5009/gnl20266.

\section{REFERENCES}

1. Renehan AG, Soerjomataram I, Tyson M, et al. Incident cancer burden attributable to excess body mass index in 30 European countries. Int J Cancer 2010;126:692-702.

2. Bardou M, Barkun AN, Martel M. Obesity and colorectal cancer. Gut 2013;62:933-947.

3. Gribovskaja-Rupp I, Kosinski L, Ludwig KA. Obesity and colorectal cancer. Clin Colon Rectal Surg 2011;24:229-243.

4. Vucenik I, Stains JP. Obesity and cancer risk: evidence, mechanisms, and recommendations. Ann N Y Acad Sci 2012;1271:37-43.

5. Otake S, Takeda H, Suzuki Y, et al. Association of visceral fat accumulation and plasma adiponectin with colorectal adenoma: evidence for participation of insulin resistance. Clin Cancer Res 2005;11:3642-3646.

6. Ibrahim MM. Subcutaneous and visceral adipose tissue: structural and functional differences. Obes Rev 2010;11:1118.

7. Després JP, Lemieux I. Abdominal obesity and metabolic syndrome. Nature 2006;444:881-887.

8. Bianchini F, Kaaks R, Vainio H. Overweight, obesity, and cancer risk. Lancet Oncol 2002;3:565-574.

9. Donohoe CL, Doyle SL, Reynolds JV. Visceral adiposity, insulin resistance and cancer risk. Diabetol Metab Syndr 2011;3:12.

10. DeFronzo RA, Ferrannini E. Insulin resistance: a multifaceted syndrome responsible for NIDDM, obesity, hypertension, dyslipidemia, and atherosclerotic cardiovascular disease. Diabetes Care 1991;14:173-194.

11. Giovannucci E. Insulin and colon cancer. Cancer Causes Control 1995;6:164-179.

12. Otani T, Iwasaki M, Sasazuki S, Inoue M, Tsugane S; Japan Public Health Center-based Prospective Study Group. Plasma C-peptide, insulin-like growth factor-I, insulin-like growth factor binding proteins and risk of colorectal cancer in a nested case-control study: the Japan public health center-based prospective study. Int J Cancer 2007;120:20072012.

13. Cecchini S, Cavazzini E, Marchesi F, Sarli L, Roncoroni L. Computed tomography volumetric fat parameters versus body mass index for predicting short-term outcomes of colon surgery. World J Surg 2011;35:415-423.

14. Goulart A, Malheiro N, Rios H, Sousa N, Leão P. Influence of visceral fat in the outcomes of colorectal cancer. Dig Surg 2019;36:33-40.

15. Hiuge-Shimizu A, Kishida K, Funahashi T, et al. Absolute value of visceral fat area measured on computed tomography scans and obesity-related cardiovascular risk factors in largescale Japanese general population (the VACATION-J study). Ann Med 2012;44:82-92.

16. Tsujinaka S, Konishi F, Kawamura YJ, et al. Visceral obesity predicts surgical outcomes after laparoscopic colectomy for sigmoid colon cancer. Dis Colon Rectum 2008;51:17571765.

17. Rickles AS, Iannuzzi JC, Mironov O, et al. Visceral obesity and colorectal cancer: are we missing the boat with BMI? J Gastrointest Surg 2013;17:133-143.

18. Clark W, Siegel EM, Chen YA, et al. Quantitative measures of visceral adiposity and body mass index in predicting rectal cancer outcomes after neoadjuvant chemoradiation. J Am Coll Surg 2013;216:1070-1081.

19. Guiu B, Petit JM, Bonnetain F, et al. Visceral fat area is an independent predictive biomarker of outcome after first-line bevacizumab-based treatment in metastatic colorectal cancer. Gut 2010;59:341-347.

20. Mahamid A, Ashkenazi I, Shapira-Rootman M, et al. Impact of increased visceral fat measured by CT on colon adenocarcinoma stage. J Gastrointest Cancer 2017;48:347-352.

21. Park SW, Lee HL, Doo EY, et al. Visceral obesity predicts fewer lymph node metastases and better overall survival in colon cancer. J Gastrointest Surg 2015;19:1513-1521.

22. WHO Expert Consultation. Appropriate body-mass index for Asian populations and its implications for policy and intervention strategies. Lancet 2004;363:157-163.

23. Matsuzawa Y, Nakamura T, Shimomura I, Kotani K. Visceral fat accumulation and cardiovascular disease. Obes Res 1995;3 Suppl 5:645S-647S.

24. Bjørndal B, Burri L, Staalesen V, Skorve J, Berge RK. Different adipose depots: their role in the development of metabolic syndrome and mitochondrial response to hypolipidemic agents. J Obes 2011;2011:490650.

25. Xiang F, Wu K, Liu Y, et al. Omental adipocytes enhance the invasiveness of gastric cancer cells by oleic acid-induced activation of the PI3K-Akt signaling pathway. Int J Biochem Cell Biol 2017;84:14-21.

26. Nieman KM, Kenny HA, Penicka CV, et al. Adipocytes promote ovarian cancer metastasis and provide energy for rapid tumor growth. Nat Med 2011;17:1498-1503.

27. Nowicka A, Marini FC, Solley TN, et al. Human omentalderived adipose stem cells increase ovarian cancer proliferation, migration, and chemoresistance. PLoS One 
2013;8:e81859.

28. Zhang Y, Nowicka A, Solley TN, et al. Stromal cells derived from visceral and obese adipose tissue promote growth of ovarian cancers. PLoS One 2015;10:e0136361.

29. McKeown-Eyssen G. Epidemiology of colorectal cancer revisited: are serum triglycerides and/or plasma glucose associated with risk? Cancer Epidemiol Biomarkers Prev 1994;3:687-695.

30. Schoen RE, Tangen CM, Kuller LH, et al. Increased blood glucose and insulin, body size, and incident colorectal cancer. J Natl Cancer Inst 1999;91:1147-1154.
31. Kaaks R, Toniolo P, Akhmedkhanov A, et al. Serum Cpeptide, insulin-like growth factor (IGF)-I, IGF-binding proteins, and colorectal cancer risk in women. J Natl Cancer Inst 2000;92:1592-1600.

32. Renehan AG, Frystyk J, Flyvbjerg A. Obesity and cancer risk: the role of the insulin-IGF axis. Trends Endocrinol Metab 2006;17:328-336.

33. Vecchié A, Dallegri F, Carbone F, et al. Obesity phenotypes and their paradoxical association with cardiovascular diseases. Eur J Intern Med 2018;48:6-17. 